\title{
Automated Visual Inspection of Moving Custom Parts
}

\author{
Shaniel Davrajh ${ }^{1}$, Thegaran Naidoo ${ }^{2}$, Glen Bright ${ }^{1}$, NS Tlale ${ }^{2}$, CM Kumile $^{3}$ \\ ${ }^{1}$ School of Mechanical Engineering, University of KwaZulu Natal, Durban, South Africa \\ ${ }^{2}$ CSIR, Pretoria, South Africa \\ ${ }^{3}$ Tshwane University of Technology, Pretoria, South Africa
}

\begin{abstract}
Mass produced custom parts require inspection routines that can facilitate variations in product parameters such as dimensions, tolerances, and throughputs. Quality control and inspection of these parts, and part families, need to occur at higher frequencies than batched produced parts. This higher frequency of inspection significantly impacts inspection times, and inherently, production rates. An effective, diverse, accurate, robust, and time efficient method for inspecting custom parts is therefore needed. Vision systems are a continuously evolving method of quality control and part inspection. These systems offer the potential to be exceptionally diverse and effective in their applications, and are therefore suited to inspecting custom parts. This paper details the research, design, construction and assembly of a prototype apparatus, which provided a suitable environment in which customized parts were inspected. System integration using the Mechatronic Engineering approach was performed to integrate vision, sensor articulation, and control systems. The apparatus was tested in a Computer Integrated Manufacturing (CIM) cell to quantify system performance. Intelligence was incorporated into the inspection routine by performing visual inspection of only significant Regions of Interest (ROI). Dynamic access by the vision sensor to the various ROIs, allowed for inspection of moving parts, which lead to an increased process efficiency. The eliminated stoppage time required by typical inspection routines, allowed for preservation of specified production rates whilst increasing frequency of inspection.
\end{abstract}

\section{INTRODUCTION}

Modern manufacturing industries are gearing their processes toward high-volume manufacturing of personalized products. This move is driven by the concept known as massPersonalization [1]. Reconfigurable Manufacturing Environments (RME) have been researched and implemented, in order to cope with these changes in customer demands of mass-producing custom parts. RME are manufacturing systems that are focused on providing rapid response to a change in product design with respect to process operations, within specified part families [2]. Changes in product design include varying throughputs, batch sizes, dimensions, colour, and assembly. The RME manufacturing strategy differs from Dedicated Manufacturing Systems (DMS), in that RME are not restricted to solely producing a single product. The difference between RME and Flexible Manufacturing Systems (FMS) is that FMS are not designed to cope with large volume production, whereas RME are able to produce small to large batch sizes. Quality control of mass-produced-custom parts involves more complex processes than inspection of standardized mass- produced parts. Custom parts are often unique in nature, and therefore require frequent inspection. If these parts are to be mass-produced, the impact of the inspection times involved on production rates must be considered. Highly flexible contact methods of inspection do not allow for hundred percent part inspections due to the high inspection times involved. Developments in vision software and computer hardware allow for fast inspection using visual methods. Machine vision systems offer the advantages of being diverse; effective; time efficient; accurate; and robust in their implementation, and are therefore suited to inspecting mass-produced custom parts. These systems are able to perform in-line inspection of parts, allowing for the detection of defective products before production of an entire batch of those products occurs. If modern manufacturers are to maintain a competitive advantage in quality control of massproduced-custom parts, they need to employ inspection routines that are frequent; cost effective; diverse; accurate; and able to provide quick response to changes in inspection requirements. Furthermore, these inspections need to be performed at rates which do not have a significant impact on production rates.

This paper details the research, design, construction, implementation, and testing of a prototype apparatus that was able to perform automated quality control with respect to inspection of mass-produced custom parts. The apparatus was referred to as the Non-Contact Automated Inspection System (NCAIS). The NCAIS was designed, using a previous Automated Visual Inspection System (AVIS) as a mechanical platform. The AVIS was responsible for performing inspection in a Computer Integrated Manufacturing (CIM) environment, in which cost was a significant factor. The Mechatronic Engineering design approach of system integration was implemented to merge sensor articulation, vision, and control subsystems of the NCAIS. The main focus of the NCAIS was to reduce inspection times initiated by requirements of frequent inspection routines of massproduced-custom parts. The apparatus was able to perform visual inspection of Regions of Interest (ROI), of moving custom parts. Intelligent path optimization for planning sensor trajectories when accessing ROI was performed. Inspection of 
moving custom parts by the NCAIS, allowed for a reduction in inspection times involved with the AVIS, allowing the NCAIS to perform inspection routines which accommodated both quantity and quality inspections. Products inspected were categorized into one of three general part families which were based on their geometries. The three part families considered were rectangular-volume parts; upright-cylindrical-volume parts; and cubic-volume parts. Template matching was performed to obtain differences between inspected parts and reference values which were based on acceptable parts. Inspection routines were able to facilitate parts that varied in throughput, dimensions, inspection requirements, and batch sizes. The NCAIS operated on the assumption of a predicable part pose throughout the inspection routine.

\section{QUALITY CONTROL OF MASS PRODUCED CUSTOM PARTS}

Quality control of products is a significant manufacturing process, when considering any manufacturing strategy []. This process may be divided into part inspection and part testing. Part inspection involves quantification of the amount that a manufactured part adheres to its design. Part testing involves relaying information as to how well a design matches an application. The advantage of performing quality control operations on products is the potential increase in customer loyalty due to sustaining production of high quality products. The disadvantage of implementing quality control processes is that time and other resources need to be invested without an increase in profit per unit, since no value is added to a product during these operations. Highly flexible inspection machines (eg. CMMs) are often employed by manufactures who wish to reduce capital investment costs by using a single machine to perform inspection of multiple parts and part families. These machines have typically high inspection times, and are not able to cope with the throughput of most production lines. Quality control using these methods needs to therefore be performed off-line. This off-line inspection facilitates inspection of only a few samples due to high inspection times involved. Consequently, defects in products may only be detected after production of a significant amount of defective parts. Batches of defective parts lead to unnecessary, and unwanted, waste of materials and production times. Inspection of mass-produced custom parts in RME needs to be performed more frequently than batch-produced parts in DMS, and at a higher rate than parts produced in FMS. The reason for requiring high frequency of inspection is due to the unique nature of the parts produced. High rates of inspection are required for reducing the impact of high frequency of inspection, on production rates. Inspection routines for RME need to facilitate changes in product parameters. Non-contact methods of inspection are preferred when inspecting massproduced custom parts due to the fact that inspection times are lower than when compared to conventional CMM methods; and inspection processes may be implemented without deformation or contamination of the inspected part occurring.
Machine vision systems were chosen for inspection of custom parts as these systems offer the capacity to perform diverse inspection in relatively short periods of time. Table 1 shows a comparison of the suitability of the different manufacturing strategies to mass-producing custom parts.

TABLE I

COMPARISON OF SUITABILITY OF MANUFACTURING SYSTEMS TO MEET REQUIREMENTS OF INSPECTING CUSTOM PARTS

\begin{tabular}{|c|c|c|c|}
\hline & DMS & FMS & RME \\
\hline \multicolumn{4}{|l|}{ Requirements } \\
\hline Production volume & High & Low & $\begin{array}{l}\text { Varying from } \\
\text { low to high }\end{array}$ \\
\hline Part variety & Very low & Vary high & $\begin{array}{l}\text { High within } \\
\text { specified part } \\
\text { families }\end{array}$ \\
\hline $\begin{array}{l}\text { Ability to facilitate } \\
\text { varying Inspection } \\
\text { requirements }\end{array}$ & Low & High & $\begin{array}{l}\text { High within } \\
\text { specified part } \\
\text { families }\end{array}$ \\
\hline $\begin{array}{l}\text { Inspection Time- } \\
\text { efficiency }\end{array}$ & $\begin{array}{l}\text { Usually } \\
\text { High }\end{array}$ & Low & High \\
\hline $\begin{array}{l}\text { Frequency of } \\
\text { inspection }\end{array}$ & Infrequent & $\begin{array}{c}\text { Very } \\
\text { frequent }\end{array}$ & $\begin{array}{l}\text { Ranges from } \\
\text { infrequent to } \\
\text { very frequent }\end{array}$ \\
\hline
\end{tabular}

Mass-produced-custom parts often only require inspection of key features in a design. An example of this requirement is the verification of fillet sizes to reduce stress concentrations on certain parts. It is possible that not all aspects of a massproduced-custom part require inspection. Inspecting only significant ROI would allow for less time being spent during the routines, and a more reliable image acquisition procedure.

\section{Mechatronic EngineERING Design}

Mechatronic engineering can be defined as the holistic design of engineering systems which involve the integration of core elements (mechanical engineering, electrical and electronic engineering with software engineering), at all levels of the design [3]. This approach involves the interchange of functionality between each of the core elements throughout the design process. The NCAIS incorporated use of the Mechatronic Engineering approach when subdividing the system into subsystems. These subsystems were the Sensor Positioning System (SPS), Vision System (VS), Materials Handling System (MHS), Part Identification System (PIS), and the software for general system management. The SPS was conceptualized to perform sensor articulation for dynamic access to various ROI. The VS was conceptualized to perform image acquisition and processing for obtaining information about the part being inspected. The PIS was responsible for identifying the part present, in order to load the necessary inspection routine parameters. The MHS consisted of a conveyor belt for moving the part through the machine; and a Part Centralization System (PCS) for aligning the centre of the part with the centre of the machine. This was performed for ensuring predictable part pose. 


\section{A. Sensor Manipulation}

The mechanical architecture of the NCAIS was based upon the design of the previous AVIS. Consequently, the spatial constraints of the new design had to match the existing platform constraints. The workspace available to the sensor manipulator was a trapezoidal volume with base $800 \mathrm{mmX} 600 \mathrm{~mm}$; top area $450 \mathrm{mmX} 600 \mathrm{~mm}$; and height $430 \mathrm{~mm}$. The mechanical design of the SPS was modeled in Solid Edge V17 to ensure correct dimensioning of components. The SPS required accurate positioning, good speed of response, and large workspace. Figure 1 below shows the assembled apparatus.

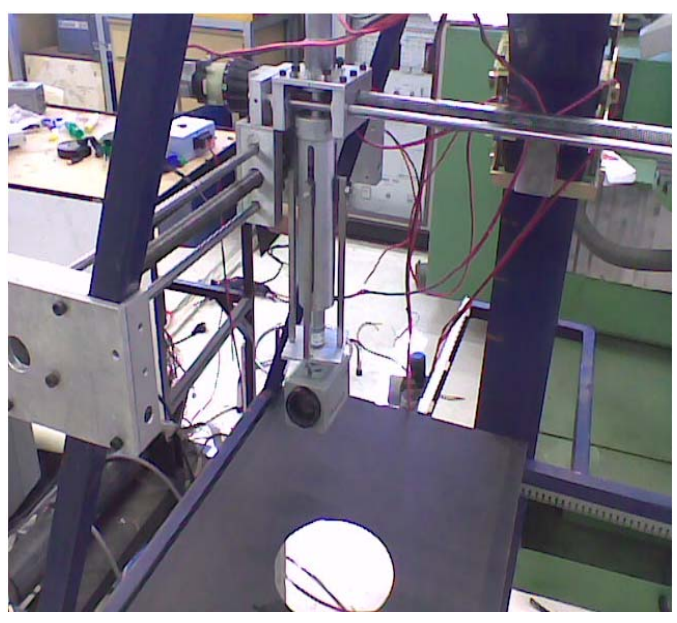

Figure 1. Assembled SPS

The selected mechanical architecture for sensor manipulation was a 3 Degree of Freedom (DOF) gantry structure, with a pan and tilt mechanism which allowed two more degrees of freedom of the sensor. The gantry structure used leadscrew drive systems for positioning the sensor, whilst the pan and tilt mechanism allowed for sensor orientation. Electric motors were used for actuation. This setup allowed for five degrees of motion.. The mechanical components of this system were modeled statically and dynamically for design verification.

A kinematic analysis of the system was performed for modeling sensor motion. This subsystem was designed to be dependant on factors relating to the Vision System. These factors included sensor Field of View (FOV); sensor-part collision detection and avoidance; and Depth of View (DOV). An analysis was performed for verification of the integrity of mechanical members under static loading conditions. Each axis of the gantry structure was analyzed independently. Essentially, the members in the $\mathrm{x}$ and $\mathrm{y}$ directions were analytically modeled as statically indeterminate beams with point loads at the leadscrew-slider interface, as shown in Figure 2.

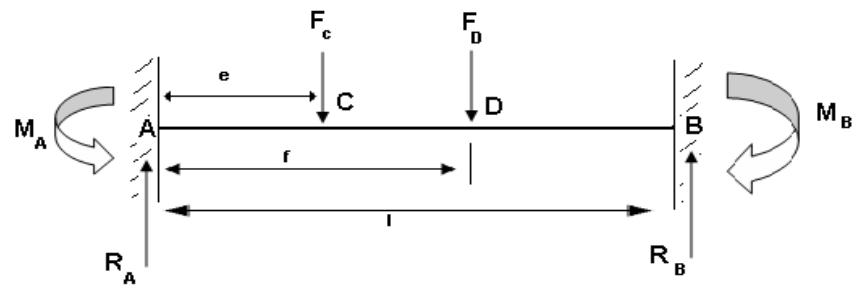

Figure2. Loading conditions assumed for analytical analyses

The deflection of these beams was calculated using equation (1) [4].

$$
v=\frac{-M_{A} x^{2}}{2 E I}+\frac{R_{A} x^{3}}{6 E I}-\frac{F_{C}(x-e)^{3}}{6 E I}-\frac{F_{D}(x-f)^{3}}{6 E I} \text { (1) }
$$

The maximum deflection was then calculated for reference against manufacturer's allowable deflections. The maximum bending moments were used to check the maximum stresses induced in the individual members, by using equation (2) [4].

$$
\sigma_{B}=\frac{M_{x} \bar{y}}{I}
$$

The motion control for sensor manipulation consisted of electrical and electronic hardware in the form of motor drivers; interfacing hardware, linear encoders; motors; and software for system control. Atmel Atmega 32 microcontrollers were selected as a master and slave motor controllers for the system. These controllers performed PWM control of the SPS, allowing for various sensor speeds during inspection routines. A dynamic analysis was performed to simulate the required response of the system under dynamic loading conditions. This was done for selecting appropriate motors by calculating the torque requirements of the system. The mean torque required for translation along each axis was given by (3) [5].

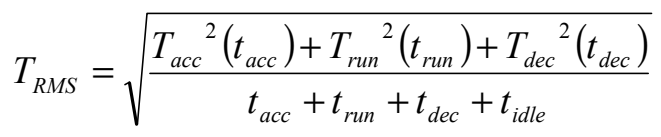

The motion of the system from one point to another was modeled as a ramp-up-ramp-down function. The kinematic model for the manipulator was designed for precisely calculating the required position and orientation of the sensor as a function of time. These positions were dependant on the ROI selected. This model had to account for the fact that the part was moving. Two sets of co-ordinate systems, namely a local and a global co-ordinate system, were defined. The local co-ordinate system originated at a point located on the moving part, and the global co-ordinate system was fixed on a point on the NCAIS. The relationship between the two co-ordinate systems was analyzed using (4), considering that the part moved in the y-direction at a constant velocity $\mathrm{y}^{\prime}$. 


$$
y(t)=y_{0}+y^{\prime} * t
$$

Mathematical relationships were established between the ROI and sensor target points. These relationships were based on the target points lying at a fixed distance from the ROI coordinates, in an outward-normal direction to the ROI. Taking

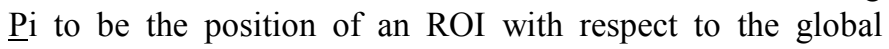
reference frame; $\mathrm{Ni}$ as the outward normal vector; and $\mathrm{d}$ as the minimum distance between the part and sensor centers, the target point was represented by

$$
\underline{T}_{i}=\underline{P}_{i}+\underline{N}_{i} d
$$

Once a set of target points for the camera was determined, an optimized path needed to be selected. The model operated on the assumption that the trajectory from one target point to the next would always be a linear path. Consequently, the method of finding the path from point to point was to subtract the linear distance of each axis of the gantry system. For $n$ inspection points, $n$ ! paths were possible. The total allowable

time $t_{\max }$ for an inspection routine was governed by the production throughput. An average camera velocity,

throughout the inspection routine, was calculated using $t_{\max }$. Referring to Figure5, the average velocity of the sensor motion throughout its journey was given by (6), with a and $b$ being the ramp-up and ramp-down times respectively.

$$
v_{\text {average }}=\frac{v_{\max }}{2 t_{\max }}\left(t_{\max }+(b-a)\right)
$$

The motion of each possible sensor path was modeled as a piecewise-linear path parametrically, as a function of time. This was used to model the camera volume as a moving sphere of radius $r$. The volumes of cubes and rectangular volumes were modeled as planes in space. The cylindrical part families were modeled using the equation of a cylinder. Solving the parametric equations, and equations of the part space simultaneously, allowed for the checking of intersection between the part space and camera volume during the inspection routine. A minimum distance $d$ was to be kept between the centers of the sensor and the part. This was performed for collision detection between sensor and part during the inspection routine. An animation of the path optimization algorithm was performed in $\mathrm{C}$, to allow for the visualization of the operation of the SPS during the inspection routine. Calculation and selection of the shortest collision-free path allowed for incorporation of some intelligence in the system design.

\section{B. Vision System}

The VS consisted of an image acquisition and image processing system. These systems were implemented using OpenCV and Matlab. The background for the system was well defined, which allowed for inspection by template matching. The reference part was passed through the machine and images of the various ROI were acquired. Prior to reference images being acquired, images of the backgrounds from the different target points were acquired. This was done to enhance the feature extraction processes, in order to locate the part. These sets of images were then stored as reference images in the system database. The image acquisition system comprised of an imaging sensor (CMOS), a lighting system, and a frame capturing software. The lighting system used was based on an illumination and colour feedback system, which allowed for variable lighting conditions [6]. The image processing software performed image enhancement; feature detection and extraction; and pixel-wise image differencing for comparing inspected parts to reference parts. Once an image was acquired, it was cropped in order to reduce the amount of information that required processing. A deconvolution operation was performed for deblurring purposes. The images were then filtered for removal of noise. This filtering process was achieved by normalizing the histograms of images. Feature detection and extraction was then executed by performing Discrete Fourier Transforms (DFT) on test images to account for translational non-idealities between the reference and test images. Log-polar operations were then performed for eliminating rotational differences between features in test and reference images. Following the different processing of images, a pixel-wise differencing algorithm was performed to determine the correlation between the test and reference images. This was performed using equation (7).

$$
\text { difference }=\left\|\frac{\sum\left(i_{\text {test }}-i_{\text {reference }}\right)}{\sum i_{\text {reference }}}\right\|
$$

The sum of the average intensities was used. A user-defined threshold was set prior to the inspection routine, in the form of a tolerance. This threshold was used to determine the acceptability of parts. For example, if the threshold was set at a value of 0.2 , and the difference between images was $30 \%$, the part was rejected. Utility programs were written for performing different operations on images. These different operations included registration of images; feature identification and extraction; and correlation checking between two images. Depending on the user defined inspection requirement, these utility files were called by the main program.

\section{SYSTEM INTEGRATION}

System integration was performed from the conceptual stages of the design till the assembly and testing phases. Matlab 7 was implemented for general system management. OpenCV was used for image capturing and processing. In order to integrate the two softwares, utility programs using OpenCV were written in C. Depending on the type of inspection and part speed required; different utilities were called from Matlab. 
For example, if a part speed was relatively low $(<1.6 \mathrm{~m} / \mathrm{min})$, the effects of blurring were negligible as they were sufficiently accommodated by the frame rate of the camera. An inspection routine with a low throughput therefore did not require deconvolution operations. The synchronization of the two softwares needed to be considered as the image capture had to occur in real-time. Integration of the mechanical and electrical designs was seen in the selection of motors used for actuation purposes; sensors used for speed and position feedback; and the hardware interfaces and motor drivers used in motion control. Integration between the VS and SPS was also required in order to perform accurate positioning of the camera along with capturing of frames, simultaneously. Integration between electronic and electrical hardware with software control systems was performed for accurate system monitoring and control of the various subsystems. Figure 2 below shows the integration of the core elements of the design.

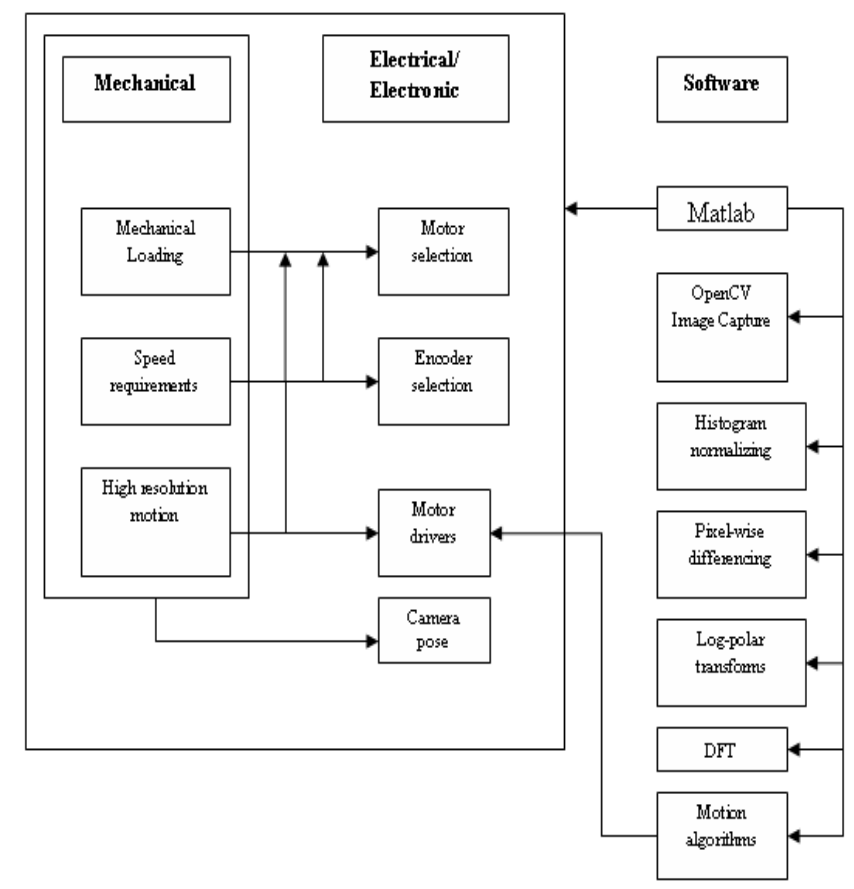

Figure.2 Integration of the core elements in the design

\section{RESULTS AND DISCUSSION}

Visual inspection of Regions of Interest (ROI) was performed on moving custom parts. This allowed for a reduction in inspection times involved with the AVIS, allowing the NCAIS to perform inspection routines which accommodated both quantity and quality inspections. Inspection of significant quantities was achieved since parts did not have to stop during inspection. The quality of the inspection routine was enhanced since large amounts of information were obtained about smaller ROI as opposed to using the same size of information to represent the part as a whole. Intelligence incorporated allowed for the optimized collision-free path to be selected whilst inspecting ROI. Inspecting only ROI allowed for images to be cropped as large amounts of information were obtained from small ROI. This meant the cropping images would not result in as significant information loss compared to if the part were inspected as a whole. Cropping the acquired images led to faster processing times.

This resulted in shorter, more detailed inspection routines. The use of template matching as a quality control mechanism was justified due to the environment being well defined. Template matching was performed to obtain differences between inspected parts and reference values which were based on acceptable parts. Any change in the inspection routine with respect to the type of inspection, did not require a new set of reference images to be acquired. Any change involving a physical change in the product however, did require a new set of reference images to be acquired, only for the relevant changes in the product.

The user was required to input dimensions, orientation of the part as it entered the machine; ROI; inspection requirements; tolerances; and throughput. Five degrees of freedom was sufficient as no sensor roll was required. Static analysis allowed for appropriate dimensioning and materials selection of mechanical members. The method of identifying the part present was a laser barcode scanner. This placed the constraint of predictable part pose on the part as it entered the machine.

The ramp-up-ramp-down model used for piecewise linear motion of the sensor allowed for accurate control, as the overshoot involved was reduced. Forward kinematics was used for the kinematic modeling of the SPS since the gantry system offered trivial solutions of point to point vector subtraction, with independent translation and rotation motions. This simplified software and hardware requirements as each motion was independent. Once a set of target points for the sensor were established, a list of possible paths was generated. Collision detection between the sensor and the part was then performed in order to eliminate unacceptable paths. The shortest path from the remaining collision-free paths was then selected as the optimal path for that particular routine. These optimal paths were dependant upon the throughput of the part; ROI that required inspection on the part; part dimensions; and part families. The camera trajectories between inspection points were all linear paths. This allowed for an average velocity to be calculated for the paths, given a maximum allowable time which was governed by product throughput. The camera space was modeled as a moving sphere with radius given by the minimum distance $d$, between the sensor and the part. This distance $\mathrm{d}$ was used to maintain a constant focus when acquiring images, allowing for reliable image acquisition process, and collision avoidance for optimal path selection. 
USB CMOS camera was chosen due to spatial and cost constraints. The images acquired were $1.3 \mathrm{MPx}$ in size. This was sufficient for assembly verification and flaw detection with respect to visible flaws. Noise in images was initiated by imperfect lighting conditions, reflections in the background of images; and vibrations imparted to the camera during image acquisition Varying the lighting conditions allowed for more reliable and consistent image acquisition process which was less prone to noisy images due to unpredictable environments. Cleaning of images using histogram normalization reduced the effect of noisy images. De-convolving operations on images were performed on routines that involved high part velocities. The log polar operations were performed in order to account for a non-accurate part positioning and orientations. The accuracy of the gantry structure allowed for precise positioning of the sensor relative to the moving part. This system was not infallible in that scaling errors were present. This could have been solved in one of two ways. The first would have been to place reference beacons around the mechanical structures. These beacons would have then been used to calculate the size of a part, or feature of a part, by using information about the current pose of the sensor relative to the global frame of reference, and the known sized of a fixed beacon.

Utility programs were written for performing different image processing tasks. The problem experienced with intergrating these softwares was that the initializing times involved with the image capturing process led to inaccurate real-time image capture. This was solved by initiating the image capturing utility program simultaneously with the main Matlab program. Matlab had image processing functions which operated at slow speed. Use of OpenCV for image capture and processing reduced the required times for full inspection significantly. The Mechatronic engineering approach was used to integrate the design of the various subsystems. The VS was required to cope with the speed of the SPS in order to make a decision before the part exited the machine. The image capturing process was based on accurate positioning of the sensor as a function of time. In order to achieve this, synchronization of the VS and SPS control systems was required. This synchronization was performed using a set counter in Matlab.

\section{CONCLUSION}

Frequent inspections of custom parts were performed. The impact of these inspections on production rates was reduced due to inspecting only significant ROI and performing these inspections on moving parts. Future work may involve use of CAD models for reference values, and multiple sensors for increasing the diversity of inspection routines.

\section{REFERENCES}

[1] http://www.nsf.gov/pubs/2000/nsf00137/nsf001371.htm,J July2007

[2] http://erc.engin.umich.edu/publications/KorenRMS.pdf, May 2007

[3] http://en.wikipedia.org/wiki/Mechatronicsechatronics ]http://www.techni on.ac. $11 / \sim$ meyoram/Lec\%2012.pdf, May 2008

[4] R C Stephens, "Mechanics of Materials", Gere and Timoshenko, Chapter 9, 2000

[5] http://www.motioncontrolonline.org/files/public/Motion_Control Basics.pdf,, February 2008

[6] www.avagotech.com/led, August 2007

[7] Eganza J, Bright G, "Dual Sensor Quality Control and Reverse Engineering System for Agile Manufacturing Systems", International Conference on Competitive Manufacturing, COMA 2007, pp 217-221, 2007 